INPLASY

PROTOCOL

To cite: Hao et al. Risk factors for tigecycline-induced hypofibrinogenaemia: A systematic review and Metaanalysis. Inplasy protocol 202220058. doi:

10.37766/inplasy2022.2.0058

Received: 15 February 2022

Published: 16 February 2022

Corresponding author: Hao Yupei

289348141@qq.com

Author Affiliation: The first hospital of hebei medical university.

Support: None.

Review Stage at time of this submission: Piloting of the study selection process.

Conflicts of interest:

None declared.

\section{Risk factors for tigecycline-induced hypofibrinogenaemia: A systematic review and Meta-analysis}

Hao, Y1; Yu, J2; Wang, L³.

Review question / Objective: Hypofibrinogenaemia is major treatment-related adverse event associated with tigecycline therapy, which in some cases can result in treatment termination. We aimed to identify the risk factors for tigecycline-induced hypofibrinogenaemia. P: Patients treated with tigecycline; I/E: Diagnosis of hypofibrinogenaemia; C:no; O:Risk factors of tigecycline induced hypofibrinogenaemia; S:case-control or cohort studies.

Condition being studied: Hypofibrinogenaemia is major treatment-related adverse event associated with tigecycline therapy, which in some cases can result in treatment termination. We aimed to identify the risk factors for tigecycline-induced hypofibrinogenaemia.

INPLASY registration number: This protocol was registered with the International Platform of Registered Systematic Review and Meta-Analysis Protocols (INPLASY) on 16 February 2022 and was last updated on 16 February 2022 (registration number INPLASY202220058).

\section{INTRODUCTION}

Review question / Objective: Hypofibrinogenaemia is major treatmentrelated adverse event associated with tigecycline therapy, which in some cases can result in treatment termination. We aimed to identify the risk factors for tigecycline-induced hypofibrinogenaemia.
P: Patients treated with tigecycline; I/E: Diagnosis of hypofibrinogenaemia; C:no; O:Risk factors of tigecycline induced hypofibrinogenaemia; S:case-control or cohort studies.

Condition being studied:

Hypofibrinogenaemia is major treatmentrelated adverse event associated with 
tigecycline therapy, which in some cases can result in treatment termination. We aimed to identify the risk factors for tigecycline-induced hypofibrinogenaemia.

\section{METHODS}

Search strategy: Search strategy: A search strategy will be developed using a combination of medical subheadings (MeSH) words and free word.TheMeSH words i n c I u d e "tigecycline","Afibrinogenemia". Taking the strategy of retrieving an PubMed database as example, the search strategy is as follows:

\#1 (Tigecycline[Title/Abstract] OR TBGMINO[Title/Abstract] OR (9-(tertButylglycylamido)minocycline) [Title/ Abstract] OR Tygacil[Title/Abstract] OR GAR 936[Title/Abstract] OR GAR-936[Title/ Abstract] OR GAR936[Title/Abstract]) OR ("Tigecycline"[Mesh])

\#2 (Afibrinogenemia[Title/Abstract] OR Afibrinogenemias[Title/Abstract] OR Congenital Afibrinogenemia[Title/Abstract] OR Afibrinogenemia, Congenital[Title/ Abstract] OR Afibrinogenemias, Congenital[Title/Abstract] OR Congenital Afibrinogenemias[Title/Abstract] OR Familial Afibrinogenemia[Title/Abstract] OR Afibrinogenemia, Familial[Title/Abstract] OR Afibrinogenemias, Familial[Title/ Abstract] OR Familial Afibrinogenemias[Title/Abstract] OR Congenital Afibrinogenaemia[Title/ Abstract] OR Afibrinogenaemia, Congenital[Title/Abstract] OR Afibrinogenaemias, Congenital[Title/ Abstract] OR Congenital Afibrinogenaemias[Title/Abstract] OR Hypofibrinogenemia, Congenital[Title/ A bstract] OR Congenita I Hypofibrinogenemia[Title/Abstract] OR Congenital Hypofibrinogenemias[Title/ Abstract] OR Hypofibrinogenemias, Congenital[Title/Abstract] OR Deficiency, Fibrinogen[Title/Abstract] OR Fibrinogen Deficiencies[Title/Abstract] OR Fibrinogen Deficiency[Title/Abstract] OR Hypofibrinogenemia[Title/Abstract] OR Hypofibrinogenemias[Title/Abstract]) OR ("Afibrinogenemia"[Mesh])
\#3 (relative[Title/Abstract] AND risk*[Title/ Abstract]) OR (relative risk[Text Word]) OR risk ${ }^{*}[$ Text Word]

\#4 \#1 and \#2 and \#3.

Participant or population: Patients treated with tigecycline.

Intervention: No.

Comparator: No.

Study designs to be included: Case-control or cohort studies.

Eligibility criteria: Eligibility criteria: Papers were screened using the following inclusion criteria:case-control studies or cohort studies on risk factors of tigecycline induced chypofibrinogenaemia.patients were assigned to the hypofibrinogenaemia group ( $<2.0 \mathrm{~g} / \mathrm{L})$ and normal fibrinogen (normal) group ( $\geq 2.0 \mathrm{~g} / \mathrm{L}$ ) to assess the clinical features of patients with t i g e c y c I in e - a s s o c i a t e d hypofibrinogenaemia. Exclusion criteria: case reports, editorials, reviews and basic studies, incomplete data that are not otherwise available, duplicated or overlapping studies, and literature that are not available for risk factors.

Information sources: The following electronic databases will be searched from the inception through the present to find studies that live up to standard: our PubMed, EMBASE, Web of Science, Cochrane Library, CNKI, Wanfang, CBM and VIP.We will also search clinicaltrials. gov. First, the first batch of documents that a meet the standards are determined through the selection of titles and abstracts, then further screening is 2 reviewers selecting by reading the full text and recording the cause of excluded literature. If 1 standard research is we will not available online, send an email to the author to get the full text or the required data.

Main outcome(s): The literature provides the influencing factors of tigecycline induced hypofibrinogenaemia; Logistic regression analysis data were provided to 
study related factors, including $O R$ and $95 \% \mathrm{Cl}$.

Quality assessment / Risk of bias analysis: The quality of each included study was assessed using the modified NewcastleOttawa scale (NOS) . Studies with an NOS score $<3$ were classified as poor-quality studies and excluded from this metaanalysis.

Strategy of data synthesis: Two software, Revman and STATA, were selected to merge the extracted data. Firstly, heterogeneity was investigated. $<50 \%$, the fixed effect model was used for metaanalysis. When the heterogeneity test results of the included studies were $\geq 50 \%$, the random-effect model was used for meta-analysis.

Subgroup analysis: Subgroup analysis is necessary according to the studies eventually included contains $>10$ articles, the Egger test will be conducted to evaluate publication bias.

Sensitivity analysis: We conduct the sensitivity analysis by excluding literature successively. When the system review.

Country(ies) involved: China.

Keywords: Hypofibrinogenaemia, Risk factors, Tigecycline.

Contributions of each author:

Author 1 - Yupei, Hao.

Author 2 - Jing, Yu.

Author 3 - Lingjiao, Wang. 